\title{
InSAR Decorrelation at $X$ Band from the Joint TanDEM-X/PAZ Constellation
}

\author{
Francescopaolo Sica, Member IEEE, Sofie Bretzke, Andrea Pulella, José-Luis Bueso-Bello, \\ Michele Martone, Pau Prats-Iraola, Senior Member IEEE, María-José González-Bonilla, \\ Michael Schmitt, Senior Member IEEE, and Paola Rizzoli
}

\begin{abstract}
Decorrelation phenomena are always present in Synthetic Aperture Radar Interferometry (InSAR). While this implies a certain level of signal degradation, decorrelation is also a characteristic of the type of imaged target itself and can therefore be seen as a source of information. In this paper we investigate InSAR decorrelation effects at $X$ band by fitting volume and temporal decorrelation trends using the unique combination of data provided by the TanDEM-X and PAZ spaceborne missions. The innovative use of this constellation allows for the acquisition of both single- and repeat-pass data at short revisit times. The concurrent availability of simultaneous acquisitions and the fine temporal resolution makes this constellation the ideal observation scenario for the study of decorrelation phenomena. Overall, we analyze five test sites, characterized by the presence of different land cover classes, and for each of them we provide volume and temporal decorrelation fitting parameters. The performed analysis gives a first insight on the potential of combining bistatic and repeat-pass InSAR acquisitions also in view of future spaceborne constellations, which could benefit from the TanDEM-X/PAZ experience.
\end{abstract}

Index Terms-Synthetic Aperture Radar, SAR, Interferometry, InSAR, coherence, decorrelation, TanDEM-X, PAZ.

\section{INTRODUCTION}

$\mathbf{S}$ YNTHETIC Aperture Radar Interferometry (InSAR) is a well-known and powerful remote sensing technique used for the retrieval of the ground topography as well as its deformation along time. The InSAR technique exploits at least two SAR images that are acquired with a given diversity, obtained by varying one or more acquisition parameters, including the acquisition time and the position of the satellites. A bistatic single-pass InSAR system is able to simultaneously acquire two images, ensuring that the changes in the scene are minimized. In this case the spatial diversity characterizing the interferometric system is well suited for the retrieval of the ground topography by performing acrosstrack interferometry. Differently, repeat-pass interferometry is ideally characterized by a temporal diversity only, which is achieved by subsequent acquisitions over the same area. In this way temporal changes can be observed, allowing for the retrieval of ground displacement. In the following, we will

F. Sica, S. Bretzke, A. Pulella, J.L. Bueso-Bello, M. Martone, P. PratsIraola, and P. Rizzoli are with the Microwaves and Radar Institute, German Aerospace Center, Münchener Str. 20, 82234 Wessling, Germany. E-mail: \{name.surname\}@dlr.de

M.J. González-Bonilla is with the Instituto Nacional de Técnica Aerospacial, 28850 Torrejón de Ardoz, Madrid, Spain. E-mail: glezbmj@inta.es

M. Schmitt is with the Signal Processing in Earth Observation group, Technical University of Munich, Arcisstr. 21, 80333 Munich, Germany. Email: m.schmitt@tum.de indicate the spatial and the temporal separation between the acquisitions as spatial and temporal baselines, respectively.

The acquisition diversity intrinsically implies a level of mismatch between the two images, which is normally referred to as decorrelation. Besides decorrelation contributions that depend on the acquisition geometry and system parameters (such as limited sensitivity, SAR ambiguities, mutual shift of the Doppler spectra, bandwidth, etc.), the dominant decorrelation contribution in single-pass across-track interferometry is related to the capability of radar waves to penetrate into volumetric targets, such as vegetation and snow- or ice-covered areas. In these cases, the recorded backscattered signal is composed by the coherent sum of multiple reflections from the considered volume which, if illuminated from slightly different satellite positions, can lead to a certain amount of volume decorrelation. Analogously, along-track interferometry suffers from temporal decorrelation caused by small changes that happen during the time interval between the two acquisitions. If correctly modeled, decorrelation effects are a valuable input for many scientific applications including land cover classification as shown in [1] and [2], or forest mapping as shown in [3] and [4]. Nevertheless, in a generic repeat-pass system, both spatial and temporal diversity are present, leading to decorrelation phenomena which cannot be independently observed.

In this paper, we analyze temporal decorrelation effects at $\mathrm{X}$ band by jointly exploiting temporal series of TanDEM-X bistatic data and repeat-pass PAZ data. This unique constellation is able to achieve short revisit times down to 4 days and, therefore, to preserve the correlation of most of the targeted land cover classes. Furthermore, thanks to the presence of both single- and repeat-pass data, we can precisely separate volume and temporal decorrelation components and, therefore, improve the retrieval of the temporal decorrelation trend.

The paper is structured as follows: Section II describes the main characteristics of the combined constellation of TanDEM-X and PAZ. Section III presents the developed methodology for the separation of the volume and temporal decorrelation for a given land cover class, Section IV introduces the utilized data sets, while Section V shows and discusses the obtained results from the experimental analysis. Finally, the conclusions and outlook are drawn in Section VI.

\section{ThE TANDEM-X/PAZ CONSTELLATION}

TerraSAR-X (TSX) is an Earth observation SAR satellite, launched in June 2007 and developed within a public-private 
partnership between the German Aerospace Center (DLR) and Airbus Defence \& Space. Together with TanDEM-X (TDX), which was launched three years later in 2010 , they operate the TanDEM-X mission (TDM) and are used together for performing high-resolution single-pass interferometric acquisitions with a resolution up to 1 meter. The main objective of this mission was the generation of a consistent global Digital Elevation Model (DEM), successfully completed in 2016 [5]. Both spacecrafts fly in a close formation with flexible interferometric baseline selection, building a highly re-configurable constellation. This is the first bistatic radar configuration in space comprising two different satellites for performing single-pass interferometry. In February 2018, the Spanish satellite PAZ was launched [6]. It is an X band SAR mission from the Spanish national Earth observation program (PNOTS) for security and defense needs and is owned and operated by Hisdesat. It is an almost twin version of the TerraSAR-X satellite and flies on the same nominal orbit as well. This allows for the acquisition of dense timeseries, obtained by combining the monostatic channel of the TanDEM-X constellation together with PAZ, allowing for the computation of repeat-pass coherences at different intervals. The three satellites PAZ, TerraSAR-X, and TanDEM-X form a highly flexible constellation with an improved acquisition capability and reduced revisit times. Particularly, the satellites have a revisit time of 11 days and are displaced over the same orbit with a 4 days separation, forming a 4+7 days constellation. As an example, Figure 1 shows all possible interferometric pairs with corresponding temporal baselines for a TDM-PAZ time-series composed by 5 acquisitions.

\section{Methodology}

In this paper, we investigate volume and temporal decorrelation effects for different types of land cover using TDM-PAZ time series. The combined use of TanDEM-X and PAZ data allows for an accurate analysis of the temporal decorrelation by achieving a denser temporal sampling, while TanDEM-X bistatic acquisitions enable the accurate modeling of the volume decorrelation at different spatial baselines. In the following, we first recall the interferometric coherence and its factorization, and afterward, we present the developed methodology.

\section{A. InSAR coherence and decorrelation phenomena}

The degree of similarity between the two images composing the interferometric pair is quantified by means of the modulus of their mutual complex correlation, namely the interferometric coherence. This parameter can be decomposed in the product of different components that depend among others on the sensor parameters, acquisition geometry, and land cover type of the observed scene. Starting from the work in [7], we factorize the total coherence $\rho_{\text {tot }}$ as:

$$
\rho_{\text {tot }}=\rho_{\text {SNR }} \rho_{\text {quant }} \rho_{\text {amb }} \rho_{\text {az }} \rho_{\text {rg }} \rho_{\text {vol }} \rho_{\text {temp }},
$$

where the terms on the right-hand side identify the coherence loss due to the limited SNR $\left(\rho_{\mathrm{SNR}}\right)$, quantization noise $\left(\rho_{\text {quant }}\right)$, ambiguities $\left(\rho_{\mathrm{amb}}\right)$, relative shift of the Doppler

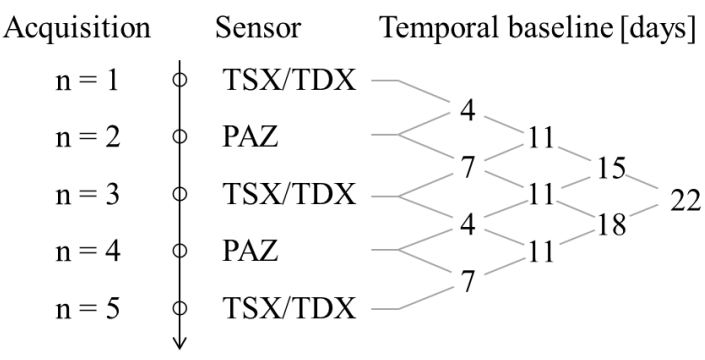

Fig. 1: Acquisition Timeline of the TDM-PAZ constellation showing the available temporal baselines.

spectra $\left(\rho_{\mathrm{az}}\right)$, baseline decorrelation $\left(\rho_{\mathrm{rg}}\right)$, volume decorrelation $\left(\rho_{\mathrm{vol}}\right)$, and temporal decorrelation $\left(\rho_{\mathrm{temp}}\right)$. The first five terms can be computed by knowing the system parameters, the backscatter intensity, and the local topography. Therefore, we compensate for these parameters and obtain the product of the volume and temporal correlation factors, indicated as:

$$
\eta=\rho_{\mathrm{vol}} \rho_{\mathrm{temp}} .
$$

In repeat-pass systems, the volume and temporal correlation factors cannot be normally distinguished. In some cases, as for the Sentinel-1 constellation, the system is designed to have a narrow orbital tube, which makes possible to neglect the volume correlation factor with respect to the temporal one [2]. On the contrary, the TDM-PAZ constellation presents large spatial baselines and the correct exploitation of time-series for the retrieval of temporal decorrelation trends is subject to a prior estimation of the amount of volume decorrelation. Therefore, in this paper, we provide a methodology for the separation of volume and temporal contributions and provide an estimation of temporal decorrelation trends for different land cover classes. On the one hand, the volume correlation factor $\rho_{\text {vol }}$ strongly depends on the spatial separation between the two satellites [8] and, specifically, on the height of ambiguity $h_{\mathrm{amb}}$, the topographic height corresponding to a complete $2 \pi$ cycle of the interferometric phase:

$$
h_{\mathrm{amb}}=\left(\lambda R \sin \left(\theta_{\mathrm{inc}}\right)\right) /\left(n B_{\perp}\right),
$$

where $\lambda$ is the wavelength, $R$ the slant range distance, $\theta_{\text {inc }}$ the incidence angle, $B_{\perp}$ the spatial normal baseline and $n$ is either equal to 1 or 2 in the bistatic and repeat-pass case, respectively. On the other hand, temporal decorrelation is caused by changes on ground that happen during the time interval between the two acquisitions. Both volume and temporal correlation are strongly influenced by the type of land cover. As an example, Figure 2 shows the TDM-PAZ total coherence over a selected patch computed at different temporal baselines, ranging from the bistatic case up to 11 days. The coherence decay is clearly visible, particularly over vegetated areas. In the present work, in order to identify the observed land cover class, we use the FROM-GLC land cover classification map [9] as reference.

\section{B. Volume and temporal decorrelation retrieval}

The first step toward the separation of volume and temporal decorrelation effects consists in the retrieval of the amount of 
TABLE I: Summary of the used TDM-PAZ InSAR time-series. LC $=$ land cover, $\theta_{\text {inc }}=$ mean incidence angle,

Platform $=\mathrm{TDM}$ or PAZ, AIID $=$ Acquisition Item ID, Date $=$ acquisition date, ${ }^{*}=\mathrm{TDM}$ acquisitions with very long temporal baselines.

\begin{tabular}{|c|c|c|c|c|c|c|c|c|c|c|c|c|c|c|}
\hline \multicolumn{3}{|c|}{$\overline{\text { Demmin }}$} & \multicolumn{3}{|c|}{ Salar de Uyuni } & \multicolumn{3}{|c|}{ Amazonas } & \multicolumn{3}{|c|}{ Skövde } & \multicolumn{3}{|c|}{ Greenland } \\
\hline \multicolumn{3}{|c|}{ LC: Crops (CRP), Grasses (GRS), Urban (URB) } & \multicolumn{3}{|c|}{ LC: Soil and rocks (SOL) } & \multicolumn{3}{|c|}{ LC: Rainforest (RFR) } & \multicolumn{3}{|c|}{ LC: Boreal forest (BRF) } & \multicolumn{3}{|c|}{ LC: Ice and snow (ICE) } \\
\hline \multicolumn{3}{|c|}{$\theta_{\text {inc }}=38^{\circ}$} & \multicolumn{3}{|c|}{$\theta_{\text {inc }}=48^{\circ}$} & \multicolumn{3}{|c|}{$\theta_{\text {inc }}=41^{\circ}$} & \multicolumn{3}{|c|}{$\theta_{\text {inc }}=34^{\circ}$} & \multicolumn{3}{|c|}{$\theta_{\text {inc }}=42^{\circ}$} \\
\hline Platform & AIID & Date & Platform & AIID & Date & Platform & AIID & Date & Platform & AIID & Date & Platform & AIID & Date \\
\hline PAZ & 15481 & 2019.09 .10 & PAZ & 18316 & 2019.11 .23 & TDM & 1600801 & 2019.05 .17 & PAZ & 12123 & 2019.05 .17 & PAZ & 14368 & 2019.08 .10 \\
\hline $\mathrm{TDM}^{*}$ & 1641717 & 2019.09.17 & TDM & 1654786 & 2019.11.30 & PAZ & 12185 & 2019.05.21 & $\mathrm{TDM}^{*}$ & 1600791 & 2019.05.24 & TDM & 1634742 & 2019.08.17 \\
\hline PAZ & 15895 & 2019.09.21 & PAZ & 18754 & 2019.12.04 & TDM & 1600800 & 2019.05 .28 & PAZ & 12394 & 2019.05 .28 & PAZ & 14749 & 2019.08 .21 \\
\hline TDM & 1641657 & 2019.09 .28 & TDM & 1654718 & 2019.12 .11 & PAZ & 12444 & 2019.06 .01 & PAZ & 15861 & 2019.09 .15 & TDM & 1634656 & 2019.08 .28 \\
\hline \multirow[t]{7}{*}{$\mathrm{TDM}^{*}$} & 1600895 & 2019.05 .19 & PAZ & 19243 & 2019.12.15 & $\mathrm{TDM}^{*}$ & 1600799 & 2019.06.08 & $\mathrm{TDM}^{*}$ & 1641694 & 2019.09 .22 & PAZ & 15129 & 2019.09 .01 \\
\hline & & & $\mathrm{TDM}^{*}$ & 1416699 & 2017.03 .26 & PAZ & 12776 & 2019.06 .12 & PAZ & 16297 & 2019.09 .26 & $\mathrm{TDM}^{*}$ & 1638751 & 2019.09 .08 \\
\hline & & & PAZ* & 12186 & 2019.05.20 & TDM & 1600798 & 2019.06.19 & & & & PAZ & 15490 & 2019.09 .12 \\
\hline & & & & & & PAZ & 13054 & 2019.06.23 & & & & TDM & 1640676 & 2019.09.19 \\
\hline & & & & & & PAZ & 15524 & 2019.09 .08 & & & & PAZ & 15906 & 2019.09 .23 \\
\hline & & & & & & $\mathrm{TDM}^{*}$ & 1641721 & 2019.09 .15 & & & & TDM & 1640756 & 2019.09 .30 \\
\hline & & & & & & PAZ & 15921 & 2019.09 .19 & & & & $\mathrm{TDM}^{*}$ & 1599773 & 2019.05 .10 \\
\hline
\end{tabular}

volume decorrelation $\rho_{\mathrm{vol}}$ at different spatial baselines and for each considered land cover class. For this purpose, we exploit TDM bistatic acquisitions, which are characterized by the absence of temporal decorrelation $\left(\rho_{\text {temp }}=1\right)$ and we isolate the $\rho_{\mathrm{vol}}$ factor by compensating for all other decorrelation sources, as done in [8]. In particular, we analyze a set of 100 TDM acquisitions available from the mission archive, which were acquired at the same mean incidence angle $\theta_{\text {inc }}$ of the corresponding TDM-PAZ InSAR time-series and with varying spatial baselines. We then estimate the coherence using a moving average filter with a $5 \times 5$ kernel and select images characterized by the presence of at least 500 samples belonging to the considered land cover class. Afterward, we compute the mean $\rho_{\text {vol }}$ per image and we fit the dependency of such volume correlation factors with respect to the height of ambiguity $h_{\mathrm{amb}}$ with an exponential function:

$$
\rho_{\mathrm{vol}}\left(h_{\mathrm{amb}}\right)=1-\alpha e^{-\frac{h_{\mathrm{amb}}}{\beta}} .
$$

This function generalizes the polynomial model in [8] for all possible $h_{\mathrm{amb}}$ values. Indeed, the second order polynomial function used in [8] can be seen as an approximation of eq. (4) in a limited $h_{\mathrm{amb}}$ interval. The estimated fitting parameters $\hat{\alpha}$ and $\hat{\beta}$ are the optimal parameters in the least square sense. Once $\rho_{\text {vol }}$ is correctly modeled from bistatic data, it can be used to compensate the $\eta$ factor in eq. (2) and isolate the temporal contribution. In this way we can accurately retrieve the temporal correlation factor $\rho_{\text {temp }}$ at a given temporal baseline $\delta t$. We are finally able to fit $\rho_{\text {temp }}(\delta t)$ for the considered land cover class by iterating this process for each of the multi-temporal interferometric pairs and then fitting the following exponential model [10]:

$$
\rho_{\text {temp }}(\delta t)=\left(1-\rho_{\mathrm{LT}}\right) e^{-\frac{\delta t}{\tau}}+\rho_{\mathrm{LT}},
$$

being $\tau$ the target decorrelation constant and $\rho_{\mathrm{LT}}$ the longterm coherence. Particularly, $\tau$ is proportional to the number of days that are required to reach the maximum decorrelation $\rho_{\mathrm{LT}}$, i.e. the remaining coherence value after long temporal baselines. The minimum length of $\delta t$ that is needed to correctly observe the long-term coherence depends on the type of land cover on ground. For example, for fast decorrelating classes, such as vegetation, a temporal baseline of a few months is sufficient, while for stable classes, such as bare soil, a baseline up to several years is needed. The estimated fitting parameters $\hat{\rho}_{\mathrm{LT}}$ and $\hat{\tau}$ are obtained as follows: $\hat{\rho}_{\mathrm{LT}}$ is retrieved

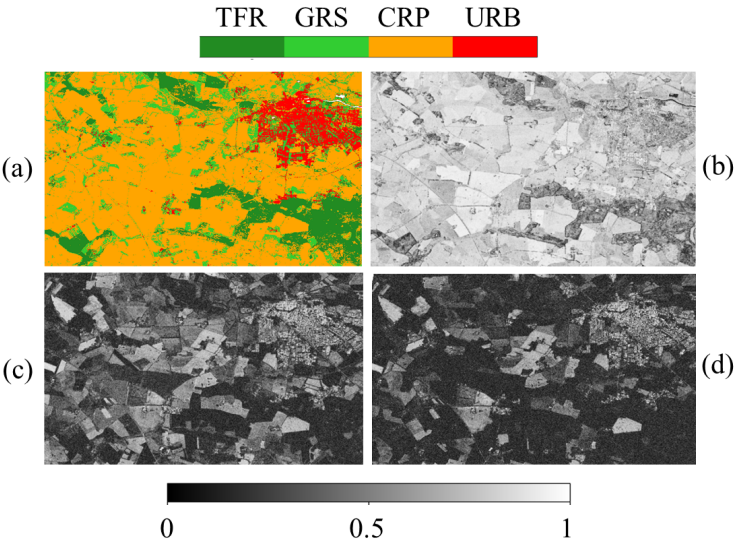

Fig. 2: (a) FROM-GLC Land cover classification map (legend: Temperate forest (TFR), Grasses (GRS), Crops (CRP), Urban areas (URB)). (b)-(d) coherence maps related to a temporal baselines $\delta t$ of (b) 0 (bistatic case), (c) 4, and (d) 11 days.

as the average coherence per class from very large temporal baseline data, while, given the estimated $\hat{\rho}_{\mathrm{LT}}, \hat{\tau}$ is the one that minimizes the fitting squared error. Note that for targets that are completely decorrelated, it is not possible to compensate for the correlation factors of eq. (1). Indeed, a thresholding operation applies for values under the coherence estimation bias, implying that eq. (1) cannot be inverted. Since we used an estimation window of 25 looks, we consider that above 0.2 the coherence estimation is bias-free. Therefore, we compensate exclusively those coherence values above 0.2 , while using the $\rho_{\text {tot }}$ instead of the $\rho_{\text {temp }}$ for the other values.

\section{MATERials}

For the present analysis we considered five test sites, each one characterized by the presence of different land cover types and located over: Demmin (Germany), Salar de Uyuni (Bolivia), Amazonas (Brazil), Skövde (Sweden), and Greenland. For each test site, we utilized TDM-PAZ interferometric timeseries in horizontal $(\mathrm{HH})$ polarization, acquired in an overall time span which goes from May 2019 up to December 2020. A summary of the used time-series, together with their main acquisition parameters and land cover classes per test site, is presented in Table I. Additionally, Figure 3 shows, for 


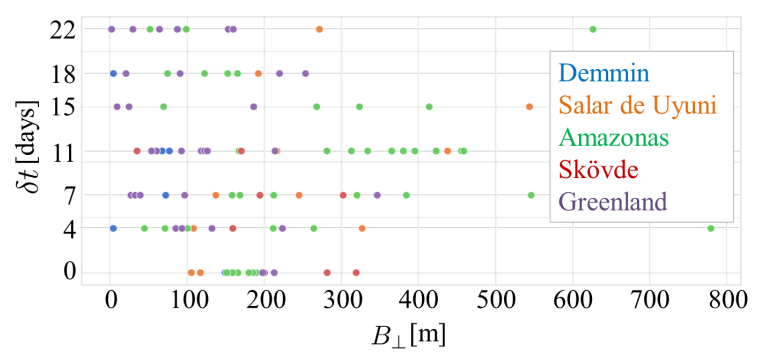

Fig. 3: Spatial baseline $B_{\perp}$ for all the considered InSAR pairs at temporal baseline $\delta t$. Each color corresponds to a different test site.

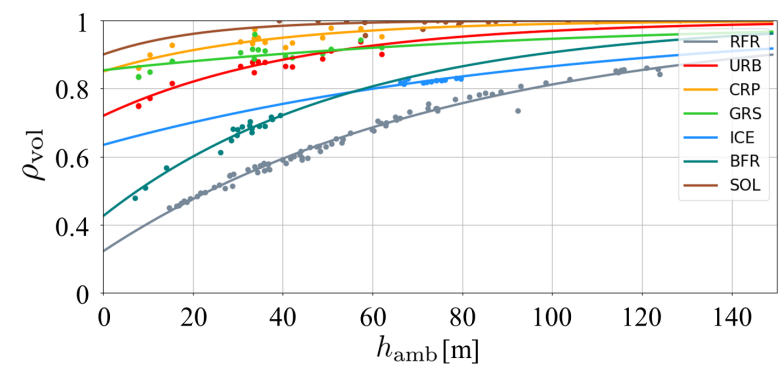

Fig. 4: Exponential fittings of $\rho_{\mathrm{vol}}$ as a function of $h_{\mathrm{amb}}$ for all the considered land cover classes (the abbreviations in the legend are defined in Table I).

each considered test site, the available $B_{\perp}$ within the timeseries with respect to the temporal baseline. Moreover, in order to retrieve the long-term coherence, we use additional TDM acquisitions with a very long temporal baseline. These acquisitions are marked with an asterisk in Table I.

\section{Results AND Discussion}

In this section, we report and discuss the results obtained from the experimental analysis introduced in Section III over the selected test sites. For all considered land cover classes, we first fitted the $\rho_{\mathrm{vol}}$ trends as a function of $h_{\mathrm{amb}}$ from archived TDM acquisitions (as explained in Section III-B), by fitting the exponential model in eq. (4). The results are shown in Figure 4 and the fitting parameters, together with the fitting root mean square error (RMSE), are summarized in Table II.

For each land cover class, the resulting fitted $\rho_{\text {temp }}$ trends are presented in Figure 5. We also fit the $\eta$ trends according to eq. (5) in order to compare its performance in terms of RMSE. For the sake of clarity, the results are split into two different subplots where, for each available temporal baseline, the corresponding $\rho_{\text {tot }}$ (triangle symbol), $\eta$ (cross symbol), and $\rho_{\text {temp }}$ (dot symbol) per image are depicted as well. From top to bottom, each subplot groups together the following land cover classes:

(a) Urban areas (URB), Soil and rocks (SOL), and Snow and ice (ICE): the first two classes are typically the most stable in time and are therefore characterized by high temporal coherence. Regarding Snow and ice, the selected test site is located in the inner part of the Greenland

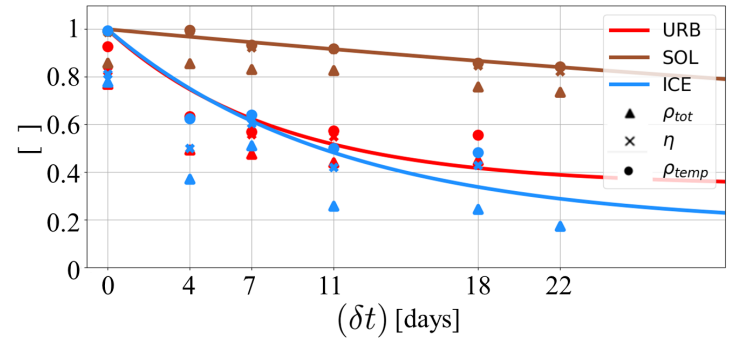

(a)

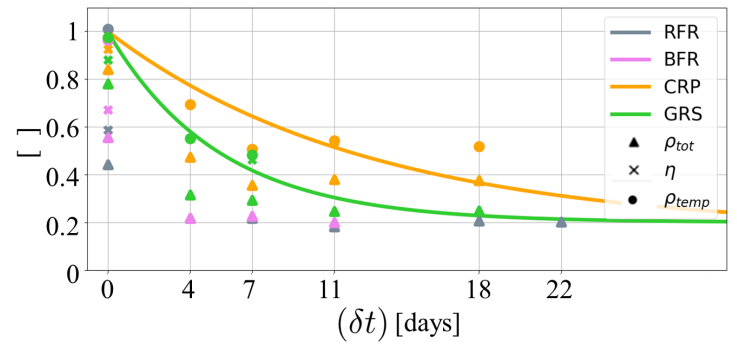

(b)

Fig. 5: Mean $\rho_{\text {tot }}, \eta$, and $\rho_{\text {temp }}$ with respect to the temporal baseline $\delta t$, for all the considered land cover classes: (a) Urban areas (URB), Soil and rocks (SOL), and Snow and ice (ICE); (b) Crops (CRP), Grasses (GRS), Rainforest (RFR), and Boreal forest (BRF). The continuous lines identify the corresponding exponential fittings of $\rho_{\text {temp }}$ using the model in (5).

plateau (acquisition center coordinates at $79^{\circ} 3^{\prime} 20^{\prime \prime} \mathrm{N}$, $33^{\circ} 24^{\prime} 39^{\prime \prime} \mathrm{W}$ ). This area is characterized by the presence of dry snow (no melting phenomena are present) and shows therefore a relatively high temporal coherence as well. Moreover, over dry snow, radar waves at $\mathrm{X}$ band penetrate inside the snow pack and are gradually absorbed with increasing depth, while only a fraction is backscattered toward the radar platform. Therefore, a significant volume decorrelation takes place as well, as clearly visible in Figure 4.

(b) Crops (CRP), Grasses (GRS), Rainforest (RFR), and Boreal forest (BFR): this plot groups together all classes characterized by the presence of some sort of vegetation. In particular, Crops and Grasses identify areas characterized by the presence of low vegetation and can show a certain variability depending on the seasonality, while Rainforest and Boreal forest correspond to two different kinds of forests. The first type is typically characterized by a tree density that is higher than the second one and both classes show significant volume decorrelation phenomena, as observed in Figure 4. This behaviour is normally caused by multiple scattering from the canopies, trunks, branches and, in presence of significant gaps, from the ground itself.

From the plots in Figure 5 and the RMSE values listed in Table II, we observe how the compensation of $\rho_{\mathrm{vol}}$ leads to a better interpretation of the temporal behavior of the imaged target. Indeed, when applying the same fitting procedure to 
TABLE II: $\rho_{\mathrm{vol}}\left(h_{\mathrm{amb}}\right), \rho_{\mathrm{temp}}(\delta t)$, and $\eta(\delta t)$ fitting coefficients. Note that the $\hat{\rho}_{\mathrm{LT}}$ is reported once since it is the same for both temporal decorrelation fittings.

\begin{tabular}{c|ccc|ccc|cc}
\hline \hline \multicolumn{8}{c}{ Fitting coefficients and RMSE } \\
\hline \hline & \multicolumn{9}{|c|}{$\rho_{\mathrm{vol}}\left(h_{\mathrm{amb}}\right)$} & \multicolumn{3}{c|}{$\rho_{\text {temp }}(\delta t)$} & \multicolumn{2}{c}{$\eta(\delta t)$} \\
\hline Land Cover & $\hat{\alpha}$ & $\hat{\beta}$ & $\mathrm{RMSE}$ & $\hat{\rho}_{\mathrm{LT}}$ & $\hat{\tau}$ & $\mathrm{RMSE}$ & $\hat{\tau}$ & $\mathrm{RMSE}$ \\
\hline Soil and rocks & 0.1 & 21.4173 & 0.0111 & 0.2007 & 103.9013 & 0.0125 & 93.4605 & 0.0132 \\
Urban areas & 0.28 & 45.0512 & 0.04 & 0.3429 & 8.2674 & 0.0661 & 7.7617 & 0.0826 \\
Crops & 0.1499 & 36.8656 & 0.0253 & 0.1658 & 12.6259 & 0.0779 & 12.4581 & 0.0781 \\
Grasses & 0.1461 & 100.01 & 0.0315 & 0.1656 & 5.3829 & 0.0326 & 4.8687 & 0.0521 \\
Rainforest & 0.6777 & 77.9722 & 0.0128 & - & - & - & - & - \\
Boreal forest & 0.5743 & 55.1436 & 0.0335 & - & - & - & - & - \\
Snow and ice & 0.3655 & 100.3 & 0.0054 & 0.1730 & 11.1726 & 0.0757 & 8.6408 & 0.1077 \\
\hline
\end{tabular}

$\eta(\delta t)$, it systematically shows a higher RMSE with respect to the $\rho_{\text {temp }}(\delta t)$. As expected, the most stable land cover classes, Soil and rocks and Urban areas at Figure 5 (a), maintain higher levels of coherence even at larger temporal baselines.

Considering now the Greenland test site in the same subplot, we can note how the targets almost completely decorrelate for circa $\delta t=30$ days and show an overall trend similar to the urban case one. Additional data show that $\rho_{\text {temp }}$ has reached the bias for $\delta t=44$ days. We omit this result in Figure 5 for the sake of the plot's clarity. Of course, it has to be pointed out that this case cannot generalize the behavior of all snowcovered regions, since such stable conditions can be found in presence of dry snow areas in Greenland or in Antarctica only, while all other snow- and ice-covered areas are typically affected by significant melting phenomena which can change the microstructure of snow and, therefore, strongly impact the coherence temporal decay.

Crops and Grasses, depicted in Figure 5 (b), decorrelate much faster than pure Soil and rocks. This is caused for example by the presence of seasonal vegetation on ground, changing soil moisture, and harvesting activities. The reader should be aware that, in our specific analysis, all available InSAR pairs were acquired during the month of September 2019 , so that it is reasonable to assume a substantial stability of the illuminated scene. Moreover, one can notice that we did not fit any exponential model to both Rainforest and Boreal forest classes. Such land cover classes completely decorrelate after a $\delta t$ which is shorter than the minimum available revisit time of 4 days. Indeed, we recorded stable values of $\rho_{\text {tot }}$ around 0.2 , independently from the considered $\delta t$ and $h_{\mathrm{amb}}$. For this reason, an exponential fitting of the temporal decay is not meaningful and we prefer to simply report the observed mean coherence values.

The $\rho_{\text {temp }}$ fitting parameters for all the available land cover classes, forests excluded, and the corresponding RMSE are summarized in Table II.

\section{CONCLUSIONS}

In the present work we exploited a unique interferometric constellation composed by the TerraSAR-X, TanDEM-X, and PAZ satellites. For the first time, a spaceborne flying formation, which is able to perform bistatic and repeat-pass InSAR acquisitions, is used for the analysis of decorrelation phenomena at $\mathrm{X}$ band. We observed the synergy and complementarity of such a constellation: on the one hand, a frequent repeat-pass represents a key asset for studying the coherence decay in time, by increasing the temporal sampling; on the other hand, the bistatic information allows for a precise compensation for the volume correlation factor, which has a strong impact on different kinds of imaged targets. Moreover, by utilizing data from the TanDEM-X mission only, this analysis would not have been possible, given the insufficient revisit time. Indeed, at $\mathrm{X}$ band, after 11 days most natural land cover classes are almost completely decorrelated. By analyzing TDM-PAZ time-series data over five different test sites, we were able to separate the volume and temporal decorrelation components for different land cover classes and analyze their behaviour. The proposed methodology has been proved by exploiting all the available TDM-PAZ time-series. Future investigations will concern a larger variety of analyzed classes as well as their variability due to seasonal effects. At the same time, the proposed work highlights the importance of combined bistatic and repeat-pass systems also in view of future SAR constellations designed for the monitoring of Earth's dynamics. Moreover, the information on temporal decorrelation can be a valuable input during the mission design, e.g., for selecting appropriate revisit times as well as for assessing the interferometric performance.

\section{ACKNOWLEDGMENT}

This research was partially funded through the HI-FIVE project, granted by the ESA Living Planet Fellowship 2018. The authors would like to thank the INTA-PAZ Science Team for providing the PAZ data (project ID: AO-001-006).

\section{REFERENCES}

[1] L. Bruzzone, M. Marconcini, U. Wertmuller, and A. Wiesmann, "An Advanced System for the Automatic Classification of Multitemporal SAR images," IEEE Transactions on Geoscience and Remote Sensing, vol. 42, no. 6, pp. 1321-1334, Jun. 2004.

[2] F. Sica, A. Pulella, M. Nannini, M. Pinheiro, and P. Rizzoli, "Repeatpass SAR interferometry for land cover classification: A methodology using Sentinel-1 Short-Time-Series," Remote Sensing of Environment, vol. 232, pp. 352-373, Oct. 2019.

[3] M. Martone, P. Rizzoli, C. Wecklich, C. Gonzalez, J.-L. Bueso-Bello, P. Valdo, D. Schulze, M. Zink, G. Krieger, and A. Moreira, "The Global Forest/Non-Forest Map from TanDEM-X Interferometric SAR Data,' Remote Sensing of Environment, vol. 205, pp. 352-373, Feb. 2018.

[4] A. Pulella, R. Aragao Santos, F. Sica, P. Posovzky, and P. Rizzoli, "Multi-Temporal Sentinel-1 Backscatter and Coherence for Rainforest Mapping," Remote Sensing, vol. 12, Mar. 2020.

[5] P. Rizzoli, M. Martone, C. Gonzalez, C. Wecklich, B. Bräutigam, D. Borla Tridon, M. Bachmann, D. Schulze, T. Fritz, M. Huber, B. Wessel, G. Krieger, M. Zink, and A. Moreira, "Generation and Performance Assessment of the Global TanDEM-X Digital Elevation Model," ISPRS Journal of Photogrammetry and Remote Sensing, vol. 132, pp. 119-139, Oct. 2017.

[6] Hisdesat, "The PAZ Mission," Apr. 2020, Available at https://www. hisdesat.es/en/paz/. Last accessed on Apr. 2020.

[7] H.A. Zebker and J. Villasenor, "Decorrelation in interferometric radar echoes," IEEE Transactions on Geoscience and Remote Sensing, vol. 30, no. 5, pp. 950-959, Sep. 1992.

[8] M. Martone, P. Rizzoli, and G. Krieger, "Volume decorrelation effects in TanDEM-X interferometric SAR data," IEEE Geoscience and Remote Sensing Letters, vol. 13, no. 12, pp. 1812-1816, Dec. 2016.

[9] P. Gong, H. Liu, and M. Zhang, "Stable classification with limited sample: transferring a 30-m resolution sample set collected in 2015 to mapping 10-m resolution global land cover in 2017,' Science Bulletin, vol. 64, pp. 370-373, Feb. 2019.

[10] F. Rocca, "Modeling interferogram stacks," IEEE Transactions on Geoscience and Remote Sensing, vol. 45, no. 10, pp. 3289-3299, Dec. 2007. 